\title{
A THEOREM ON PLANAR GRAPHS
}

BY

W. T. TUTTE

1. Introduction. In this paper we generalize the well-known theorem of Hassler Whitney on Hamiltonian circuits of triangulations [2]. The methods used are essentially the same as those of [2], but have been modified in detail so as to be applicable to all planar graphs. The generalized theorem (Theorem I below) asserts that any planar graph having a circuit has one satisfying certain specified conditions. For an important class of planar graphs, which includes those studied by Hassler Whitney, these conditions require that the circuit be Hamiltonian.

In the remainder of this introductory section we give formal definitions of some of the terms to be used in the paper and then make a precise statement of the main theorem.

A planar graph is a finite set of simple closed arcs, called edges, in the 2sphere such that any point of intersection of two distinct members of the set is an end of both of them. The vertices of a planar graph are the ends of its edges. Clearly any subset of a planar graph is a planar graph.

In accordance with this definition we say that two planar graphs meet if they have a common edge and are disjoint if they have no common edge. Two disjoint planar graphs may have one or more common vertices.

Let $G$ be any planar graph. We denote the number of its vertices by $\alpha_{0}(G)$ and the number of its edges by $\alpha_{1}(G)$. An edge $A$ and a vertex $a$ of $G$ are incident if $a$ is an end of $A$. The degree $d(a)$ of a vertex $a$ is the number of edges of $G$ incident with $a$.

The complex $|G|$ of $G$ is the union of the edges of $G$. A planar graph is a circuit if its complex is a simple closed curve, and an arc-graph if its complex is a simple arc. In the latter case we refer to the ends of the simple arc also as the ends of the arc-graph and call the other vertices of the arc-graph its internal vertices. By a circuit or arc-graph of $G$ we mean a subset of $G$ which is a circuit or arc-graph respectively. A circuit of $G$ is Hamiltonian if it has each vertex of $G$ as a vertex. An edge of $G$ which is not a member of any circuit of $G$ is an isthmus of $G$.

The vertices of attachment of a subset $H$ of $G$ are the common vertices of $H$ and $G-H$. We denote their number by $w(H)$ and call it the attachmentnumber of $H$. Clearly $w(G-H)=w(H)$.

Let $J$ be a circuit of $G$. A subset $H$ of $G-J$ is $J$-bounded if all its points of attachment are vertices of $J$. We find that if $G-J$ is non-null it has a unique

Received by the editors July 1,1955 . 
expression as a union of disjoint minimal non-null $J$-bounded subsets of $G-J$. These subsets of $G-J$ are the bridges of $J$ in $G$.

The complex $|J|$ of a circuit $J$ of $G$ is a simple closed curve. We refer to the residual domains of this curve in the 2 -sphere also as the residual domains of $J$, and say that $J$ is the bounding circuit of each of them.

Let $E$ be an edge of $G$ not an isthmus and let $D$ be a residual domain of a circuit $J$ of $G$ such that $E \in J$. Then $D$ is a terminal domain of $E$ if no circuit of $G$ containing $E$ has a residual domain which is a proper subset of $D$. If $D$ is a terminal domain of $E$ then $J$ is a terminal circuit of $E$. We find that $E$ has just two terminal domains. The corresponding terminal circuits may or may not be distinct.

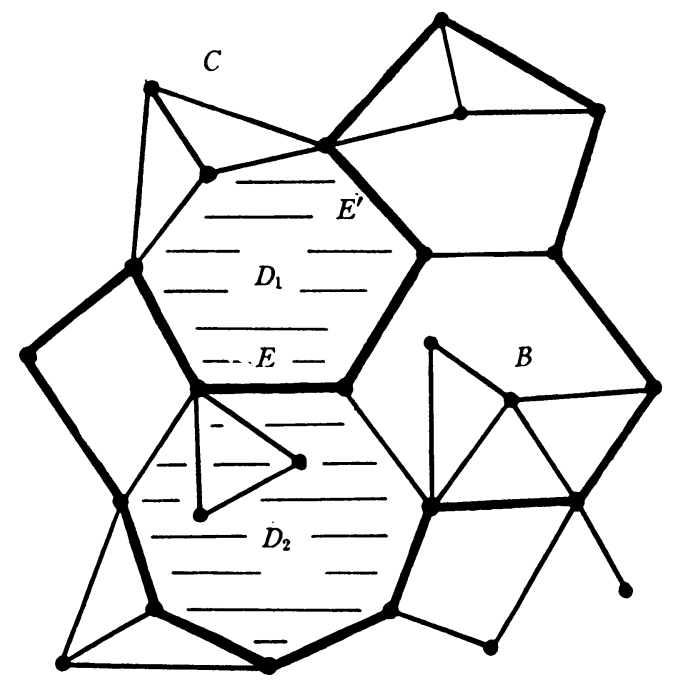

FIG. I

The main theorem of this paper is as follows.

THEOREM I. Let $G$ be any planar graph. Let $E$ be an edge of $G$ not an isthmus and let $E^{\prime}$ be an edge distinct from $E$ of a terminal circuit of $E$.

Then there exists a circuit $J$ of $G$ having the following properties:

(i) $\left(E, E^{\prime}\right) \in J$.

(ii) If $B$ is a bridge of $J$ in $G$ then $w(B) \leqq 3$.

(iii) If $B$ is a bridge of $J$ in $G$ which meets a terminal circuit of $E$ then $w(B)=2$.

We illustrate this theorem in Figure I. There the terminal domains of $E$ are the shaded regions $D_{1}$ and $D_{2}^{\prime}$. The thick lines of the diagram define a circuit $J$ satisfying conditions (i), (ii) and (iii). The letter B indicates a bridge of $J$ having attachment-number 3 and not meeting a terminal circuit of $E$. 
At $C$ we have a bridge of $J$ which does meet a terminal circuit of $E$, and which accordingly has the attachment-number 2 .

2. Bridges. Let $J$ be a circuit of a planar graph $G$.

From the definition of a $J$-bounded subset of $G-J$ we readily deduce the following propositions: $G-J$ is $J$-bounded, the complement in $G-J$ of any $J$-bounded subset of $G-J$ is $J$-bounded, and any intersection of $J$-bounded subsets of $G-J$ is $J$-bounded.

We define a bridge of $J$ in $G$ as a minimal non-null $J$-bounded subset of $G-J$. If $A \in G-J$ then the intersection of all $J$-bounded subsets of $G-J$ containing $A$ is a bridge of $J$ in $G$ containing $A$, as is easily verified from the propositions on $J$-bounded sets noted above. Hence if $G-J$ is non-null it is the union of the bridges of $J$ in $G$. Moreover two distinct bridges of $J$ in $G$ are disjoint since their intersection is $J$-bounded. Hence the bridges of $J$ have the properties specified in the Introduction.

(2.1) Let $x$ and $y$ be distinct vertices of a bridge $B$ of $J$ in $G$. Then there is an arc-graph of $B$ whose ends are $x$ and $y$ and which has no internal vertex in common with $J$.

Proof. Let $H$ be the union of all arc-graphs of $B$ having $x$ as one end and having no internal vertex in common with $J$. Then $H$ is non-null since $x$ is incident with at least one edge of $B$. Clearly $x$ can be a vertex of attachment of $H$ only if it is a vertex of $J$.

Let $z$ be any vertex of attachment of $H$ distinct from $x$. Let $C$ be any edge of $B-H$ incident with $z$ and let $t$ be the other end of $C$. There must be an arcgraph $L$ of $H$ with ends $x$ and $z$ which has no internal vertex in common with $J$. Now $t$ is not $x$ since the arc-graph $\{C\}$ is not contained in $H$. Moreover $t$ is not an internal vertex of $L$. For otherwise if $M$ is the set of all edges of $L$ between $x$ and $t$ then $M \cup\{C\}$ is an arc-graph of $H$, contrary to the definition of $C$. Hence $L \cup\{C\}$ is an arc-graph. Since it is not a subset of $H$ it follows that $z$ is a vertex of $J$.

We deduce that $H$ is $J$-bounded. Since it is non-null it is therefore identical with $B$. Hence $y$ is a vertex of $H$. The theorem follows.

(2.2) Let $L$ be an arc-graph of $G-J$ having no internal vertex in common with $J$. Then $L$ is a subset of some bridge of $J$ in $G$.

Proof. Enumerate the edges of $L$, in the order of their occurrence in the arc $|L|$, as $A(1), \cdots, A(k)$. For $1 \leqq i \leqq k$ let $B(i)$ be the bridge of $J$ containing $A(i)$. If $1 \leqq i<k$ then $B(i)$ and $B(i+1)$ are identical since they have a common vertex which is not a vertex of $J$. The theorem follows.

(2.3) If $B$ is a bridge of $J$ in $G$ then $|B|$ meets just one of the residual domains of $J$.

Proof. Let the residual domains of $J$ be $D$ and $D^{\prime}$. Assume $|B|$ meets both. Since $|B|$ meets $D$ some edge $A$ of $B$ meets $D$. This edge must lie entirely in $D$ save possibly for one or both of its ends. If both ends of $A$ are vertices of $J$ then $\{A\}$ is $J$-bounded, $B=\{A\}$ and $|B| \cap D^{\prime}$ is null. We 
deduce that $B$ has a vertex $x$ in $D$. Similarly it has a vertex $x^{\prime}$ in $D^{\prime}$. Any simple arc in $|B|$ with ends $x$ and $x^{\prime}$ must meet $|J|$. But this is contrary to (2.1). The theorem follows.

If $B$ is a bridge of $J$ and $|B|$ meets the residual domain $D$ of $J$ then we say that $B$ is a bridge over $D$.

(2.4) Let $J^{\prime}$ be a circuit of $G$ having a residual domain $D^{\prime}$ which is a subset of a residual domain $D$ of $J$. Let $B$ be a bridge of $J$ over $D$ such that some edge $A$ of $B$ meets $D^{\prime}$. Then there exists a bridge $B^{\prime}$ of $J^{\prime}$ over $D^{\prime}$ such that $A \in B^{\prime} \subseteq B$.

Proof. Let $X$ be the set of all edges of $B$ which meet $D^{\prime}$. Since $D^{\prime}$ does not meet $|J|$ the vertices of attachment of $X$ are all points of $\left|J^{\prime}\right|$, that is $X$ is $J^{\prime}$-bounded. Let $B^{\prime}$ be the bridge of $J^{\prime}$ containing $A$. Then $B^{\prime}$ is over $D^{\prime}$. Moreover $B^{\prime} \cap X$ is non-null and $J^{\prime}$-bounded and is therefore identical with $B^{\prime}$. Hence $A \in B^{\prime} \subseteq B$.

COROLlaRy. If in addition all the common vertices of $B$ and $J^{\prime}$ are vertices of $J$ then $B$ is itself a bridge of $J^{\prime}$ over $D^{\prime}$.

For then $B^{\prime}$ is $J$-bounded and therefore identical with $B$.

3. Multiple connection. A planar graph $G$ is $n$-separable, where $n$ is any non-negative integer, if it can be partitioned into two disjoint subsets $H$ and $G-H$, each having a vertex which is not a vertex of the other, such that $w(H)=w(G-H) \leqq n$. The graph $G$ is $n$-connected if it is not $m$-separable for any non-negative integer $m<n$. Thus if $j>k \geqq 0$ and $G$ is $j$-connected then $G$ is also $k$-connected. An $n$-separable planar graph has two vertices separated by not more than $n$ others and an $n$-connected one has no two vertices separated by fewer than $n$ others.

(3.1) Every circuit is 2-connected.

For a simple closed curve is connected, and its connection is not destroyed by the removal of any one point.

(3.2) Let $J$ be a circuit of a planar graph $G$ and let $B$ be a bridge of $J$ in $G$. Suppose there is a circuit $K$ of $G$ which meets both $J$ and $B$. Then $w(B) \geqq 2$.

Proof. $B \cap K$ and $(G-B) \cap K$ are complementary non-null subsets of $K$. Since $K$ is 2-connected they have at least two common vertices. But these are vertices of attachment of $B$. Hence $w(B) \geqq 2$.

(3.3) A 2-connected planar graph $G$ such that $\alpha_{1}(G) \geqq 2$ has no isthmus.

Proof. Suppose $A$, with ends $a$ and $b$, is an isthmus of $G$. Since $G$ is not 0 -separable we may suppose $d(a) \geqq 2$. Let $H$ be the union of all the arc-graphs of $G$ which have $a$ as an end and do not contain $A$. Then $H$ is non-null since $d(a) \geqq 2$. It is clear that $H$ and $G-H$ have no common vertices other than $a$ and $b$. Hence, since $G$ is not 1-separable, they have both these vertices in common. It follows that there is an arc-graph $L$ of $H$ with ends $a$ and $b$. Then $L \cup\{A\}$ is a circuit of $G$, contrary to the definition of $A$.

(3.4) If $G$ is an $n$-connected planar graph such that $\alpha_{0}(G)>n$ then $d(a) \geqq n$ for each vertex $a$ of $G$. 
Proof. Suppose $G$ has a vertex $a$ such that $d(a)<n$. Let $H$ be the set of all edges of $G$ incident with $a$. Then $a$ is a vertex of $H$ but not of $G-H$. On the other hand $\alpha_{0}(H) \leqq d(a)+1$ and therefore $G-H$ has a vertex which is not a vertex of $H$. But $w(H)=w(G-H) \leqq d(a)<n$, contrary to the hypothesis that $G$ is $n$-connected.

(3.5) Let $B$ be a bridge of a circuit $J$ of an n-connected planar graph $G$. Then if $w(B)<n$ one of the following alternatives is true:

(i) $B$ has just one edge and both ends of this edge are vertices of $J$.

(ii) $\alpha_{1}(J)=w(B)$ and $B$ is the only bridge of $J$ in $G$ having more than one edge.

Proof. It may happen that both ends of some edge $A$ of $B$ are vertices of $J$. Then $\{A\}$ is $J$-bounded and so $B=\{A\}$. In this case alternative (i) holds. In the remaining case $B$ has a vertex which is not a vertex of attachment. Since $w(B)<n$ the graph $G$ is not $w(B)$-separated and therefore the only vertices of $G-B$ are the vertices of attachment of $B$. This implies that $J$ has just $w(B)$ vertices and therefore just $w(B)$ edges. Hence $n>w(B) \geqq 2$ and so by (3.4) $B$ has at least two edges. No bridge of $J$ other than $B$ can have more than one edge, for such a bridge would have a vertex not a vertex of attachment of $B$.

4. Terminal domains. Let $G$ be any non-null planar graph.

An arc-graph $L$ of $G$ is joinable if there is a circuit $J$ of $G$ such that $L \subset J$. Thus if $E \in G$ the arc-graph $\{E\}$ is joinable if and only if $E$ is not an isthmus of $G$.

Let $L$ be a joinable arc-graph of $G$ and let $J$ be a circuit of $G$ such that $L \subset J$. Let the residual domains of $J$ be $D$ and $D^{\prime}$. We call $D$ a terminal domain of $L$ in $G$ if there is no circuit $K$ of $G$ containing $L$ such that some residual domain of $K$ is a proper subset of $D$. If either $D$ or $D^{\prime}$ is a terminal domain of $L$ in $G$ we call $J$ a terminal circuit of $L$ in $G$. Thus if $E$ is an edge of $G$ which is not an isthmus the terminal domains and circuits of $\{E\}$ agree respectively with the terminal domains and circuits of the edge $E$ as defined in the Introduction.

If $H$ is a subset of $G$ and $K$ is a subset of $G-H$ we write $p(H, K)$ for the number of vertices of $H$ which are vertices of $K$, and $q(H, K)$ for the number of vertices of attachment of $H$ which are not vertices of $K$. Thus $p(H, K)$ $+q(H, K)=w(H)$.

If $Q$ is any circuit of $G$ we define a transversal of $Q$ as an arc-graph $M$ of $G-Q$ such that the ends of $M$, but no other vertices of $M$, are vertices of $Q$. If $M$ satisfies this condition it is a subset of some bridge $B$ of $Q$, by (2.2). If $B$ is over the residual domain $D$ of $Q$ we say $M$ is a transversal of $Q$ over $D$. It then follows from (2.3) that $|M|$ is a simple arc whose ends are in $|Q|$ but which otherwise lies entirely in $D$, that is $|M|$ is a cross-cut in $D$.

(4.1) Let $L$ be a joinable arc-graph of $G, D$ a terminal domain of $L$, and $J$ the corresponding terminal circuit of $L$. Let $B$ be any bridge of $J$ over $D$. Then $p(B, J-L) \leqq 1$. 
Proof. Suppose $p(B, J-L)>1$. Then there are two distinct common vertices $x$ and $y$ of $B$ and $J$ which are not internal vertices of $L$. By (2.1) there is a transversal $M$ of $J$ over $D$ with ends $x$ and $y$. But there is an arc-graph $N$ of $J$ with ends $x$ and $y$ such that $L \subseteq N$. Clearly $M \cup N$ is a circuit of $G$ containing $L$ and one of its residual domains is a proper subset of $D$. This contradicts the definition of $D$.

(4.2) A joinable arc-graph $L$ of $G$ has just two terminal domains in $G$. Moreover if $J$ is any circuit of $G$ which contains $L$ and has residual domains $D$ and $D^{\prime}$, then one terminal domain of $L$ is a subset of $D$ and the other is a subset of $D^{\prime}$.

Proof. A circuit $J$ of $G$ containing $L$ exists. If its residual domains are $D$ and $D^{\prime}$ then, since $G$ is finite, it follows from the definition of a terminal domain that there are terminal domains $T$ and $T^{\prime}$ of $L$ contained in $D$ and $D^{\prime}$ respectively. As $T$ and $T^{\prime}$ occupy opposite sides of the arc $|L|$ any third terminal domain $T^{\prime \prime}$ of $L$ must meet either $T$ or $T^{\prime}$.

Suppose $T^{\prime \prime}$ meets $T$. Let $K$ and $K^{\prime \prime}$ be the bounding circuits of $T$ and $T^{\prime \prime}$ respectively. If $K^{\prime \prime}$ meets a bridge $B$ of $K$ over $T$ then the non-null planar graphs $K^{\prime \prime} \cap B$ and $K^{\prime \prime} \cap(G-B)$ have at least two common vertices since $K^{\prime \prime}$ is 2 -connected by (3.1). Since $L \subset K^{\prime \prime}$ neither of these is an internal vertex of $L$. Hence $p(B, K-L) \geqq 2$, contrary to $(4.1)$.

We deduce that $\left|K^{\prime \prime}\right|$ does not meet $T$. Hence $T$ is a subset of a residual domain of $K^{\prime \prime}$. This residual domain must be $T^{\prime \prime}$ since $T^{\prime \prime}$ meets $T$. Thus $T \subseteq T^{\prime \prime}$ and therefore $T^{\prime \prime}=T$, since $T^{\prime \prime}$ is a terminal domain of $L$. A similar argument shows that if $T^{\prime \prime}$ meets $T^{\prime}$ then $T^{\prime \prime}=T^{\prime}$. This completes the proof of the theorem.

If $G$ is 2-connected then each edge of $G$ has two distinct terminal domains, by (3.3) and (4.2), provided that $\alpha_{1}(G) \geqq 2$. The terminal domains of the edges of $G$ do not meet $|G|$, by (4.1). They may readily be identified as the components of the complement of $|G|$ in the 2 -sphere. These components are of ten called the countries or regions of the map determined by $G$.

(4.3) Let $B$ be a bridge over a residual domain $D$ of a circuit $J$ of $G$. Let $x$ and $y$ be distinct vertices of attachment of $B$ and let $L$ be an arc-graph of $J$ with ends $x$ and $y$. Let $T$ be the terminal domain of $L$ contained in $D$. Then there is a transversal $M$ of $J$ over $D$, with ends $x$ and $y$, such that $L \cup M$ is the bounding circuit of $T$.

Proof. By (2.1) there is a transversal $N$ of $J$ over $D$ with ends $x$ and $y$. Then $L \cup N$ is a circuit of $G$ with a residual domain $D^{\prime}$ contained in $D$. By (4.2), $T \subseteq D^{\prime}$. Now it is clear that the bounding circuit of $T$ may be written as $L \cup M$, where $M$ is an arc-graph of $G$ with ends $x$ and $y$. Since $T \subseteq D^{\prime}$ an internal vertex of $M$ is either an internal vertex of $N$ or a point of $D^{\prime}$. It is therefore not a vertex of $J$. Moreover $|M|$ does not meet the residual domain of $J$ other than $D$. Hence $M$ is a transversal of $J$ over $D$.

Consider a bridge $B$ of a circuit $Q$ of $G$ such that $w(B) \geqq 2$. Let the vertices of attachment of $B$ be $a_{1}, a_{2}, \cdots, a_{k}$. These vertices separate $Q$ into $k=w(B)$ 
disjoint arc-graphs $S_{1}, S_{2}, \cdots, S_{k}$. We can adjust the notation so that the ends of $S_{i}$ are $a_{i}$ and $a_{i+1}$ for each $i$ (with $a_{k+1}=a_{1}$ ). We call the arc-graphs $S_{i}$ the segments of $Q$ determined by $B$.

(4.4) Let $B$ and $B^{\prime}$ be bridges of a circuit $J$ of $G$ over the same residual domain $D$ of $J$. Suppose $w(B) \geqq 2$. Then there is a segment of $J$ determined by $B$ whose vertices include all the vertices of attachment of $B^{\prime}$.

Proof. We may suppose $w\left(B^{\prime}\right) \geqq 2$, for otherwise the theorem is trivial.

Suppose first that $B^{\prime}$ has a vertex of attachment $b$ which is an internal vertex of some segment $S_{i}$ of $J$ determined by $B$. Assume that $B^{\prime}$ also has a vertex of attachment $c$ which is not a vertex of $S_{i}$. Then $B^{\prime}$ contains a transversal $M^{\prime}$ of $J$ over $D$ with ends $b$ and $c$, by (2.1). Similarly $B$ contains a transversal $M$ of $J$ over $D$ whose ends are the ends $a_{i}$ and $a_{i+1}$ of $S_{i}$. The two arcs $|M|$ and $\left|M^{\prime}\right|$ have a common point in $D$. This is impossible since $B$ and $B^{\prime}$ are distinct. So in this case the theorem is true.

In the remaining case each vertex of attachment of $B^{\prime}$ is a vertex of attachment of $B$. Then we may suppose $w(B) \geqq 3$, since otherwise the theorem is trivial. Let $b$ be a vertex of attachment of $B^{\prime}, U$ and $V$ the segments of $J$ determined by $B$ which have $b$ as an end, and $a$ and $c$ the other ends of $U$ and $V$ respectively. Then $a, b$, and $c$ are distinct since $w(B) \geqq 3$.

Let $F$ be the terminal domain contained in $D$ of the joinable arc-graph $U \cup V$. By (4.3) its bounding circuit is of the form $U \cup V \cup M$, where $M$ is a transversal of $J$ over $D$ with ends $a$ and $c$. The cross-cut $|M|$ separates $D$ into two simply connected domains $F$ and $F^{\prime}$ whose bounding circuits are $U \cup V \cup M$ and $(J-(U \cup V)) \cup M$ respectively. Both $|B|$ and $\left|B^{\prime}\right|$ meet $F$ since $b$ is a boundary point of $F$ but not of $F^{\prime}$.

By (2.2) $M$ is a subset of some bridge $C$ of $J$ over $D$. We have $C=B$. For otherwise, by the corollary to (2.4), $B$ is a bridge over $F$ violating (4.1). But now (4.1) and the Corollary to (2.4) imply that $B^{\prime}$ is a bridge over $F$ not having both $a$ and $c$ as vertices of attachment. So by the definition of $B^{\prime}$ its vertices of attachment are either the ends of $U$ or the ends of $V$.

(4.5) Let $L$ be a joinable arc-graph of $G$ with ends $a$ and $b$, and let $E$ be the edge of $L$ incident with $a$. Let $D$ be a terminal domain of $L$ and $D^{\prime}$ the terminal domain of $E$ contained in $D$. Let $J$ and $J^{\prime}$ be the bounding circuits of $D$ and $D^{\prime}$ respectively. Then there is at most one bridge $B$ over $D$ which meets $J^{\prime}$ and satisfies $p(B, J-L)>0$.

Proof. If $x$ and $y$ are distinct vertices of $J$ we denote by $N(x, y)$ the arcgraph of $J$ which has $x$ and $y$ as ends and includes $E$.

Let $U$ denote the class of all bridges $B$ over $D$ which meet $J^{\prime}$ and satisfy $p(B, J-L)>0$. If $B \in U$ then by (3.2) and (4.1) we have also $q(B, J-L)>0$.

If $U$ is non-null we find, using (2.1), that there exist vertices $x$ and $y$ of $J$ with the following properties:

(i) $x$ is a vertex of $J-L$,

(ii) $y$ is an internal vertex of $L$, 
(iii) there is a transversal $M(x, y)$ of $J$ over $D$ with ends $x$ and $y$,

(iv) $\alpha_{1}(N(x, y))$ has the least value consistent with (i), (ii), and (iii).

Let $D(x, y)$ be the terminal domain of $N(x, y)$ contained in $D$. By (4.2) we have $D^{\prime} \subseteq D(x, y)$. By (2.2) and (4.3) we may suppose the transversal $M(x, y)$ chosen so that the bounding circuit of $D(x, y)$ is $M(x, y) \cup N(x, y)$. Let $C$ be the bridge over $D$ having $M(x, y)$ as a subset.

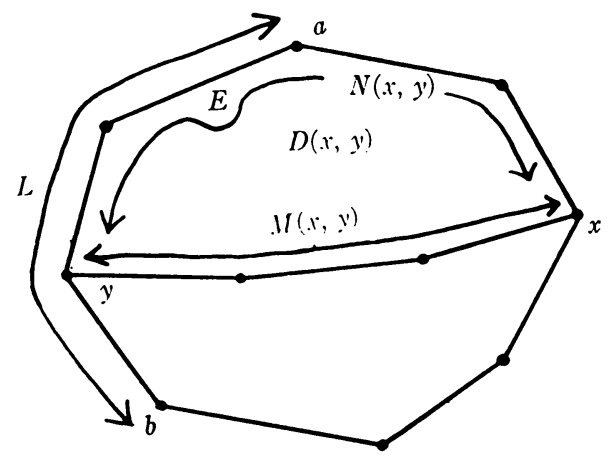

FIG. II

Suppose $B \in U$ and that $B \neq C$. Then $B$ includes an edge of $G$ meeting $D(x, y)$, since $B$ meets $J^{\prime}$ and $D^{\prime} \subseteq D(x, y)$. Hence $B$ is a bridge over $D(x, y)$, by the corollary to (2.4). Since $p(B, J-L)>0$ and $q(B, J-L)>0$ it follows from (2.1) and the choice of $x$ and $y$ that $x$ and $y$ are both vertices of $B$. But this is contrary to (4.1). It follows that $C$ is the only member of $U$.

5. Proof of Theorem I. For planar graphs in which each edge is an isthmus Theorem I is without content. It is convenient to say that it is trivially true for such planar graphs.

We denote by $X$ the class of all planar graphs for which Theorem I is true. We write $Y$ for the class of all planar graphs $G$ such that $H \in X$ whenever $H$ is a planar graph having fewer edges than $G$. We note that $G \in X$ if $G$ is a planar graph satisfying $\alpha_{1}(G) \leqq 1$.

If $G$ is a planar graph, $L$ a joinable arc-graph of $G$, and $E$ an edge of $G-L$, we denote by $V(G, L, E)$ the class of all circuits $J$ of $G$ having the following properties:

(i) $L \cup\{E\} \subseteq J$,

(ii) If $B$ is a bridge of $J$ then $p(B, J-L) \leqq 3$,

(iii) If $B$ is a bridge of $J$ meeting a terminal circuit of $L$ then $p(B, J-L)$ $=2$.

(5.1) Suppose $G \in Y$. Let $L$ be a joinable arc-graph of $G$ satisfying $\alpha_{1}(L) \geqq 2$, $K$ a terminal circuit of $L$, and $E$ an edge of $K-L$. Then $V(G, L, E)$ is non-null.

Proof. Let the terminal domains of $L$ be $D$ and $D^{\prime}$, with bounding circuits $K$ and $K^{\prime}$ respectively. 
Let $S$ be the set of all edges of $G-L$ having an internal vertex of $L$ as one end. We obtain a planar graph $H$ from $G-S$ by replacing the members of $L$ by the arc $|L|$, considered as a single edge. The same operation converts any circuit $Q$ of $G$ such that $L \subseteq Q$ into a circuit $O(Q)$ of $H$ such that $|O(Q)|=|Q|$ and therefore $|L| \in O(Q)$. It is clear that any circuit of $H$ which has $|L|$ as an edge is of the form $O(Q)$, where $Q$ is a circuit of $G$ such that $L \subseteq Q$. It follows that the terminal domains of $|L|$ in $H$ are $D$ and $D^{\prime}$ and that the corresponding terminal circuits of $|L|$ are $O(K)$ and $O\left(K^{\prime}\right)$ respectively.

Now $\alpha_{1}(H)<\alpha_{1}(G)$ since $\alpha_{1}(L) \geqq 2$, and therefore $H \in X$. Applying Theorem I to $H$ we find that there is a circuit $J$ of $G$ containing $L$ such that $O(J) \in V(H,\{|L|\}, E)$.

Let $B$ be any bridge of $J$ in $G$. If $B \subseteq S$ then $B$ has at most one vertex not an internal vertex of $L$, by (2.1). In the remaining case we write $B^{\prime}$ for the non-null set $B-(B \cap S)$. This set is a bridge of $O(J)$ in $H$. For any subset $C$ of $B^{\prime}$ which is $O(J)$-bounded in $H$ gives rise to a subset of $B$ which is $J$ bounded in $G$ when we adjoin to it those members of $S$ which are incident with vertices of $C$ not vertices of $J$. Since $O(J) \in V(H,\{|L|\}, E)$ we have

(i) $w\left(B^{\prime}\right) \leqq 3$ in $H$,

(ii) $w\left(B^{\prime}\right)=2$ in $H$ if $B^{\prime}$ meets $O(K)$ or $O\left(K^{\prime}\right)$.

Since neither $K$ nor $K^{\prime}$ meets $S$ we deduce that in each case $p(B, J-L) \leqq 3$ in $G$ and further that $p(B, J-L)=2$ in $G$ if $B$ meets $K$ or $K^{\prime}$. But $(|L|, E)$ $\in O(J)$ and therefore $L \cup\{E\} \subseteq J$. It follows that $J \in V(G, L, E)$.

(5.2) $Y \subseteq X$.

Proof. Let $G$ be any member of $Y, E$ any edge of $G$ not an isthmus, and $E^{\prime}$ any edge distinct from $E$ of some terminal circuit of $E$. We denote the terminal domains of $E$ by $D_{1}$ and $D_{2}$ and the corresponding terminal circuits of $E$ by $K_{1}$ and $K_{2}$ respectively, adjusting the notation so that $E^{\prime} \in K_{1}$.

Suppose $\left\{E, E^{\prime}\right\}$ is a circuit $J$ of $G$. Then $J$ has just two vertices and so $w(B) \leqq 2$ for each bridge $B$ of $J$. Using (3.2) we deduce that $J \in V(G,\{E\}$, $\left.E^{\prime}\right)$. Thus Theorem I holds in this case.

In the remaining case $K_{1}$ has at least three edges. Hence we can find an arc-graph $L$ of $K_{1}$ with ends $a$ and $a^{\prime}$ having the following properties:

(i) $\left(E, E^{\prime}\right) \in L$,

(ii) $a$ is an end of $E$ but not of $E^{\prime}$, and $a^{\prime}$ is an end of $E^{\prime}$ but not of $E$. (See Figure III.)

Let the terminal domains of $L$ be $D_{3}$ and $D_{4}$. By (4.2) we can adjust the notation so that $D_{1} \subseteq D_{3}$ and $D_{2} \subseteq D_{4}$. But since $L \subseteq K_{1}$ we have, also by (4.2), $D_{3} \subseteq D_{1}$. Hence $D_{1}$ and $D_{3}$ are identical. We denote the bounding circuit of $D_{4}$ by $K_{4}$. In Figure III, $D_{4}$ is the shaded region.

We select an edge $E^{\prime \prime}$ of $K_{4}-L$ according to the following rules. If there is a bridge $B$ over $D_{1}$ which meets $K_{2}$ and has a vertex in common with $K_{4}-L$, then by (4.5) there is only one such bridge. Moreover by (4.1) $B$ has 


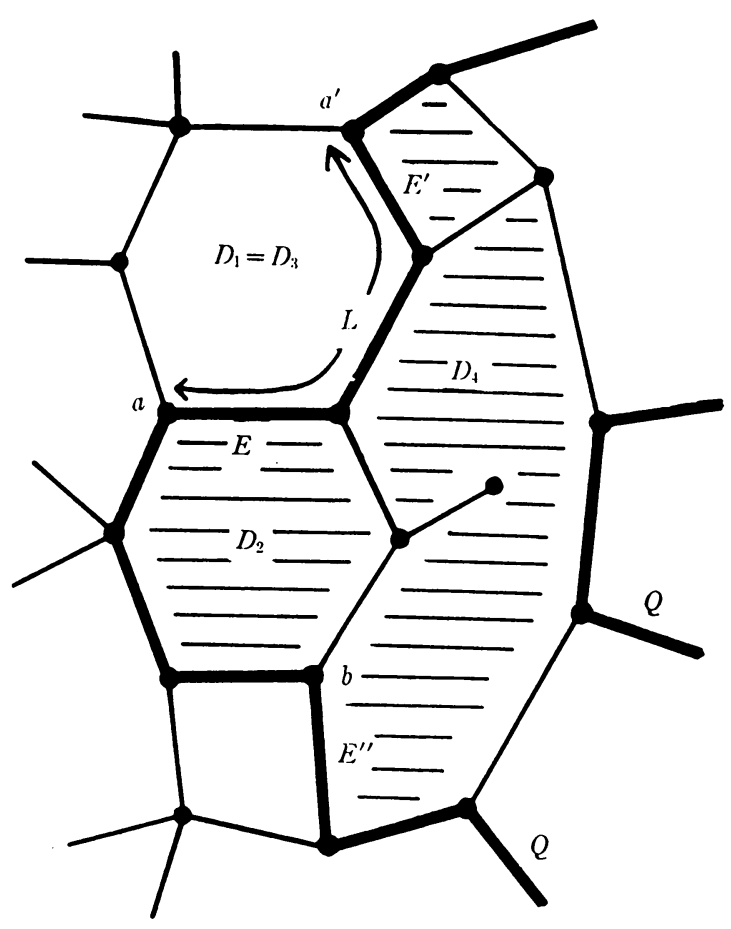

FIG. III

only one vertex, $b$ say, in common with $K_{4}-L$. In this case we choose $E^{\prime \prime}$ as an edge of $K_{4}-L$ incident with $b$. If there is no such bridge $B$ any member of $K_{4}-L$ may be taken as $E^{\prime \prime}$.

By (5.1) there is a circuit $Q$ of $G$ such that $Q \in V\left(G, L, E^{\prime \prime}\right)$. We denote the residual domains of $Q$.by $R_{1}$ and $R_{2}$, adjusting the notation so that $D_{1} \subseteq R_{1}$ and $D_{2} \subseteq D_{4} \subseteq R_{2}$. (See (4.2).)

A bridge $B$ of $Q$ in $G$ is singular if it satisfies one of the following conditions:

(i) $w(B)>3$,

(ii) $B$ meets $K_{1}$ or $K_{2}$, and $w(B) \neq 2$. In the latter case we have $w(B)>2$, by (3.1).

Lemma I. $A$ singular bridge $B$ of $Q$ is a bridge over $R_{2}$. It satisfies $p(B$, $Q-L) \leqq 2$ and $q(B, Q-L) \geqq 2$. Moreover if it meets $K_{2}$ it satisfies $p(B, Q-L)$ $\leqq 1$.

Proof. We discuss first the case in which $B \cap K_{2}$ has an edge not belonging to $K_{4}$. Then $B$ is over $R_{2}$, since it meets $K_{2}$. By (2.4) there is a bridge $B^{\prime}$ over $D_{4}$ which meets $K_{2}$ and satisfies $B^{\prime} \subseteq B$. Now $p\left(B^{\prime}, K_{4}-L\right)=0$ or 1 , by (4.1). 
Then by the choice of $E^{\prime \prime}$ any common vertex of $B^{\prime}$ and $K_{4}-L$ is a vertex of $Q$. So in each case $B^{\prime}$ is $Q$-bounded and therefore identical with $B$. Hence $p(B, Q-L) \leqq 1$. Since $B$ is singular it follows that $w(B) \geqq 3$ and $q(B, Q-L) \geqq 2$.

In the remaining case $B$ meets $K_{4}$ if it meets $K_{2}$. So by the definitions of $Q$ and $B$ the relations $w(B)>3$ and $p(B, Q-L) \leqq 3$ hold if $B$ does not meet $K_{1}$ or $K_{4}$, and the relations $w(B)>2$ and $p(B, Q-L)=2$ hold if $B$ meets $K_{1}$ or $K_{4}$. In each case $B$ has a vertex $x$ which is an internal vertex of $L$. Let $A$ be an edge of $B$ incident with $x$.

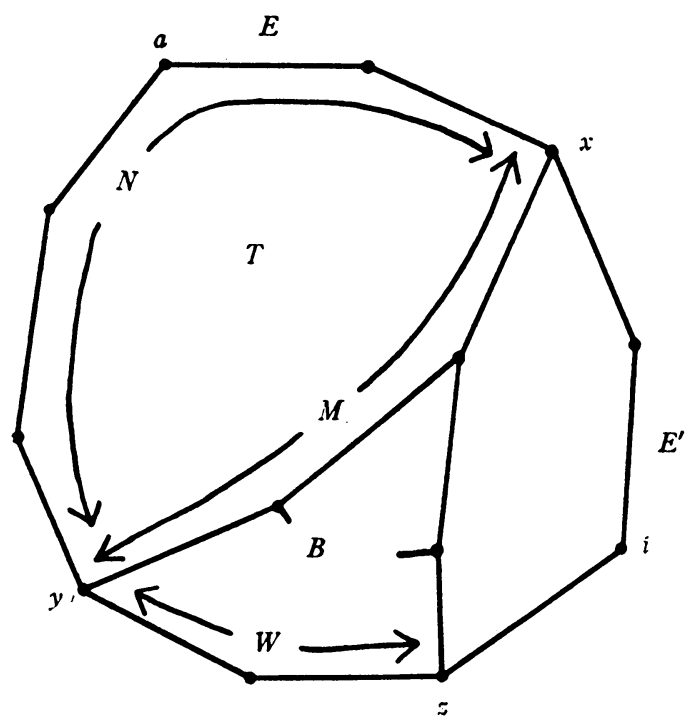

FIG. IV

Suppose $B$ is over $R_{1}$. Then $A$ meets $D_{1}$. By (2.4) there is a bridge $B_{1}$ over $D_{1}$ such that $A \in B_{1} \subseteq B$. By (4.1), $w\left(B_{1}\right)=1$. Hence $B_{1}$ is $Q$-bounded and therefore identical with $B$. But this is impossible since $w(B) \geqq 3$. We deduce that $B$ is over $R_{\mathbf{2}}$.

If $B$ meets $K_{2}$ we may now suppose $B \cap K_{2} \subseteq K_{4}$. The argument used in this case is illustrated by Figure IV, in which the outer circuit represents $K_{4}$. There must be an arc-graph $W$ of $B \cap K_{2}$ whose ends, $y$ and $z$ say, are vertices of attachment of $B$. We adjust the notation so that either $y=a$ or $y$ separates $a$ and $z$ in $\left|K_{4}-L\right|$. Let $N$ be the arc-graph of $K_{4}$ with ends $x$ and $y$ which includes $E$. By (2.1) there is a transversal $M$ of $K_{4}$ over $D_{4}$ with ends $x$ and $y$. Let $T$ be the residual domain of the circuit $M \cup N$ contained in $D_{1}$. Then $D_{2} \subseteq T$, by (4.2). But this is impossible since the boundary point $z$ of $D_{2}$ belongs neither to $T$ nor to its boundary $M \cup N$.

Last, suppose $B$ does not meet $K_{2}$. As $A$ meets $D_{4}$ there is a bridge $B^{\prime}$ over

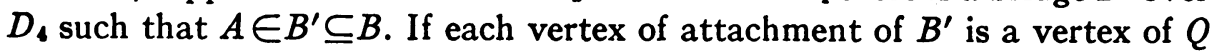


we find that $B^{\prime}=B$. Then $p(B, Q-L)=p\left(B^{\prime}, K_{4}-L\right) \leqq 1$ by (4.1). Since $w(B) \geqq 3$ we then have $q(B, Q-L) \geqq 2$. If instead $B^{\prime}$ has a vertex of attachment $z$ which is not a vertex of $Q$ then $B$ includes the two edges of $K_{4}$ incident with $z$. So in this case $p(B, Q-L)=2$. But $w(B)>3$ since $B$ does not meet $K_{2}$. Hence $q(B, Q-L) \geqq 2$. This completes the proof of the lemma.

Let $B$ be any singular bridge of $Q$. By Lemma I, $q(B, Q-L) \geqq 2$. Hence there is a unique arc-graph $L(B)$ of $L-\left\{E, E^{\prime}\right\}$ whose ends are vertices of $B$ and whose vertices include all vertices of $B$ which are internal vertices of $L$. Clearly $L(B)$ is a union of segments of $Q$ determined by $B$. We say that a bridge $B^{\prime}$ of $Q$ distinct from $B$ is enclosed by $B$ if one of its vertices is an internal vertex of $L(B)$.

If $B^{\prime}$ is enclosed by $B$ then all the vertices of attachment of $B^{\prime}$ are vertices of $L(B)$. If $B^{\prime}$ is over $R_{2}$ this is a consequence of (4.4). If $B^{\prime}$ is over $R_{1}$ we prove it as follows. There is an edge $A$ of $B^{\prime}$ incident with an internal vertex $x$ of $L(B)$. This edge must meet $D_{1}$. By (2.4) there is a bridge $B^{\prime \prime}$ over $D_{1}$ such that $A \in B^{\prime \prime} \subseteq B^{\prime}$. By (4.1) $x$ is the only vertex of attachment of $B^{\prime \prime}$. But then $B^{\prime \prime}$ is $Q$-bounded and so identical with $B^{\prime}$.

If $B$ encloses another singular bridge $B^{\prime}$ then $B^{\prime}$ does not enclose $B$. For otherwise the above results, in conjunction with (4.4) would make $L(B)$ identical with $L\left(B^{\prime}\right)$ and a segment of $Q$ determined by both $B$ and $B^{\prime}$.

We deduce that there exists a set $B=\left\{B_{1}, \cdots, B_{k}\right\}$ of singular bridges of $Q$ such that no member of $B$ encloses another and each singular bridge of $Q$ is enclosed by some member of $B$.

For each $B_{i} \in B$ we define the corresponding singularity $Z_{i}$ of $Q$ as the union of $B_{i}, L\left(B_{i}\right)$ and all the bridges of $Q$ enclosed by $B_{i}$.

The singularities $Z_{i}$ and $Z_{j}$ corresponding to two distinct members $B_{i}$ and $B_{j}$ of $B$ have no edge in common. For suppose they have a common edge $A$. If $A \notin L$ then the bridge of $Q$ containing $A$ must be enclosed by both $B_{i}$ and $B_{j}$. In this case some internal vertex of $L\left(B_{i}\right)$ is a vertex of $L\left(B_{j}\right)$ and so these two arc-graphs have a common edge. We may therefore suppose $A \in L\left(B_{i}\right)$ $\cap L\left(B_{j}\right)$. Since neither of $B_{i}$ and $B_{j}$ encloses the other the arc-graphs $L\left(B_{i}\right)$ and $L\left(B_{j}\right)$ have the same ends and are therefore identical. Moreover $L\left(B_{i}\right)$ is a segment of $Q$ determined by both $B_{i}$ and $B_{j}$. If $w\left(B_{i}\right)>2$ this segment includes as vertices all the vertices of attachment of $B_{j}$, by (4.4), and therefore $w\left(B_{j}\right)=2$. This contradicts our assumption that $B_{i}$ and $B_{j}$ are both singular bridges.

For each $B_{i} \in B$ any edge of $G$ incident with an internal vertex of $L\left(B_{i}\right)$ is necessarily an edge of $Z_{i}$. Hence the vertices of attachment of $Z_{i}$ are the common vertices of $B_{i}$ and $Q-L\left(B_{i}\right)$. We define the norm $Z$ of $Q$ as the set of all edges of $G-Q$ which do not belong to any singularity of $Q$. Each vertex of attachment of $Z$ is a vertex of $Q$ not an internal vertex of any of the arcgraphs $L\left(B_{i}\right)$.

For each singularity $Z_{i}$ we write $G_{i}=Q \cup Z_{i}$. Since $G_{i} \subseteq G$ we have $G_{i} \in Y$. 
Lemma II. Each $G_{i}$ has a circuit $Q_{i}$ with the following properties:

(i) $Q-L\left(B_{i}\right) \subseteq Q_{i}$,

(ii) If $C$ is a bridge of $Q_{i}$ in $G_{i}$ then $w(C) \leqq 3$. Moreover if $C$ meets $K_{1}$ or $K_{2}$ then $w(C)=2$.

Proof. We denote the ends of $L\left(B_{i}\right)$ by $b$ and $b^{\prime}$ in such a way that $b$ separates $b^{\prime}$ from the end $a$ in the arc-graph $L$. We write $\bar{L}=Q-L\left(B_{i}\right)$ and denote by $U$ the edge of $\bar{L}$ incident with $b$.

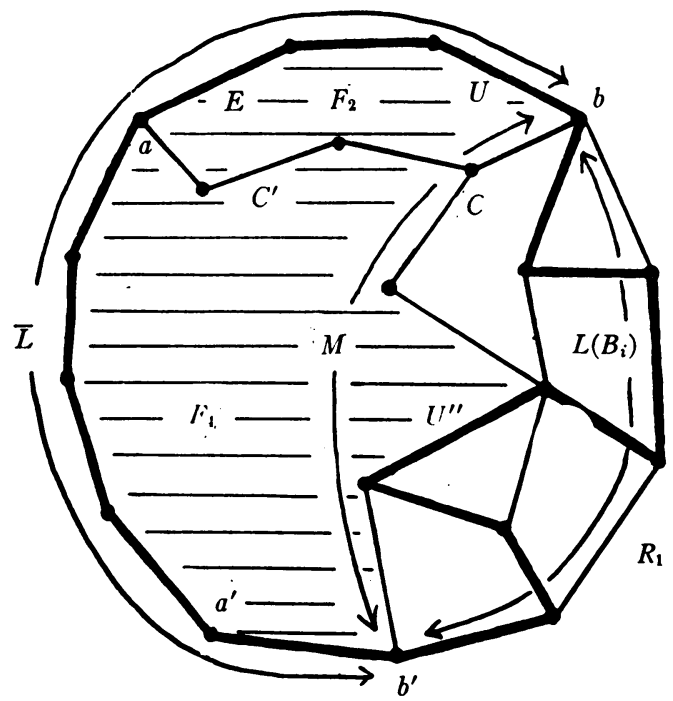

FIG. V

The only bridges over $R_{1}$ in $G_{i}$ are the bridges over $\dot{R}_{1}$ in $G$ which are enclosed by $B_{i}$. We have seen that each such bridge has attachment-number 1 . Hence by (3.2) no circuit of $G_{i}$ containing $U$ has an edge meeting $R_{1}$. It follows that $R_{1}$ is a terminal domain of $U$ and of $\bar{L}$ in $G_{i}$. Let the terminal domains of $U$ and $\bar{L}$ contained in $R_{2}$ be $F_{2}$ and $F_{4}$ respectively and let their bounding circuits be $P_{2}$ and $P_{4}$ respectively. By (4.2) we have $D_{2} \subseteq F_{2}$ since $P_{2}$ must include all the edges of $L$ between $a$ and $b$. In Figure $\mathrm{V}$ the shaded region represents $F_{4}$.

By (4.3), $P_{4}=\bar{L} \cup M$, where $M$ is a transversal of $Q$ over $R_{2}$ with ends $b$ and $b^{\prime}$. Since $\alpha_{1}(\bar{L}) \geqq 2$ it follows from (5.1) that for each edge $U^{\prime \prime}$ of $M$ we can find a circuit $Q_{i}$ of $G_{i}$ having the following properties.

$$
Q-L\left(B_{i}\right) \subseteq Q_{i} \text {. }
$$

If $C$ is a bridge of $Q_{i}$ in $G_{i}$ then $p\left(C, Q_{i}-\bar{L}\right) \leqq 3$.

Moreover if $C$ meets $Q$ or $P_{4}$ then $p\left(C, Q_{i}-\bar{L}\right)=2$. 


$$
U^{\prime \prime} \in Q_{i}
$$

In Figure $\mathrm{V}$ the thick lines form a circuit satisfying these conditions.

We shall show that we can always choose $U^{\prime \prime}$ so that $Q_{i}$ necessarily satisfies condition (ii) of the lemma. First however we establish some properties of $Q_{i}$ which hold for all choices of $U^{\prime \prime}$.

We denote the residual domains of $Q_{i}$ by $S_{1}$ and $S_{2}$ so that $R_{1} \subseteq S_{1}$ and $S_{2} \subseteq R_{2}$. We have $F_{4} \subseteq S_{2}$, by (4.2).

Let $C$ be any bridge of $Q_{i}$ in $G_{i}$. If it meets $K_{2}$ then since $D_{2} \subseteq F_{4}$ it meets either $P_{4}$ or a bridge $C^{\prime}$ over $F_{1}$ in $G_{i}$. In the latter case $C^{\prime}$ has at most one vertex of attachment which is not an internal vertex of $\bar{L}$, by (4.1). But if $w\left(C^{\prime}\right) \leqq 1$ in $G_{i}$ it is clear that $w\left(C^{\prime}\right) \leqq 1$ in $G$, which is contrary to (3.2). Hence $C$ meets either $P_{4}$ or a bridge $C^{\prime}$ over $F_{4}$ in $G_{i}$ such that $w\left(C^{\prime}\right) \geqq 2$ and at least one vertex of attachment of $C^{\prime}$ is an internal vertex of $\bar{L}$. In the latter case we must have $C^{\prime} \subseteq C$.

A similar argument in which $\bar{L}, F_{4}$ and $P_{4}$ are replaced by $\{U\}, F_{2}$ and $P_{2}$ respectively shows that if $C$ meets $K_{2}$ it also meets $P_{2}$. Analogously if $C$ meets $K_{1}$ it also meets $Q$.

If no vertex of $C$ is an internal vertex of $\bar{L}$ we have $p\left(C, Q_{i}-\bar{L}\right)=w(C)$. Then by (2) and the above results $C$ must satisfy condition (ii).

Suppose $C$ is over $S_{1}$ and has an internal vertex of $\bar{L}$ as a vertex. It does not meet $K_{2}$ since $D_{2} \subseteq S_{2}$. It has an edge $A$ incident with an internal vertex of $\bar{L}$. This edge must meet $R_{1}$. So by (2.4) there is a bridge $C^{\prime}$ over $R_{1}$ such that $A \in C^{\prime} \subseteq C$. Then $w\left(C^{\prime}\right)=1$ by (4.1). Hence $C^{\prime}$ is $Q_{i}$-bounded and therefore $C=C^{\prime}$. Thus $w(C)=1$. Further $C$ does not meet $Q$ and therefore does not meet $K_{1}$. Hence it satisfies (ii).

It remains only to show that we can choose $U^{\prime \prime}$ so that (ii) is satisfied whenever $C$ is a bridge over $S_{2}$ satisfying $q\left(C, Q_{i}-\bar{L}\right) \geqq 1$.

Suppose $p\left(B_{i}, Q-L\right)=0$. Then there is no bridge $C$ over $Q_{i}$ in $G_{i}$ satisfying $q\left(C, Q_{i}-\bar{L}\right) \geqq 1$ for any choice of $U^{\prime \prime}$. Hence (ii) is satisfied for any choice of $U^{\prime \prime}$.

Next suppose $p\left(B_{i}, Q-L\right)=1$. We denote the common vertex of $B_{i}$ and $Q-L$ by $x$. It is the only internal vertex of $\bar{L}$ which is a vertex of $Z_{i}$. It separates $\bar{L}$ into two disjoint arc-graphs, $\bar{L}_{1}$ with ends $b$ and $x$ and $\bar{L}_{2}$ with ends $x$ and $b^{\prime}$. (See Figure VI.)

Now all circuits of $G_{i}$ having $U$ as an edge must contain $\bar{L}_{1}$. Hence the terminal domain of $\bar{L}_{1}$ contained in $R_{2}$ is $F_{2}$. By (4.3) we have $P_{2}=L_{1} \cup M^{\prime}$, where $M^{\prime}$ is a transversal of $Q$ over $R_{2}$ with ends $b$ and $x$. Let $y$ be the last vertex of $M^{\prime}$, counting from $b$, which is a vertex of $M$, and let $T$ be the arcgraph of $M^{\prime}$ with ends $x$ and $y$. Then $T$ is a transversal of $P_{4}$ over $F_{4}$. By (2.2) it is a subset of a bridge $C^{\prime \prime}$ of $P_{4}$ over $F_{1}$ in $G_{i}$. By (4.1) $y$ is the only common vertex of $C^{\prime \prime}$ and $M$. Thus $w\left(C^{\prime \prime}\right)=2$. Further $y$ is an internal vertex of $M$. For otherwise $C^{\prime \prime}$ would be a bridge of $Q$ in $G$, distinct from $B_{i}$ and not 


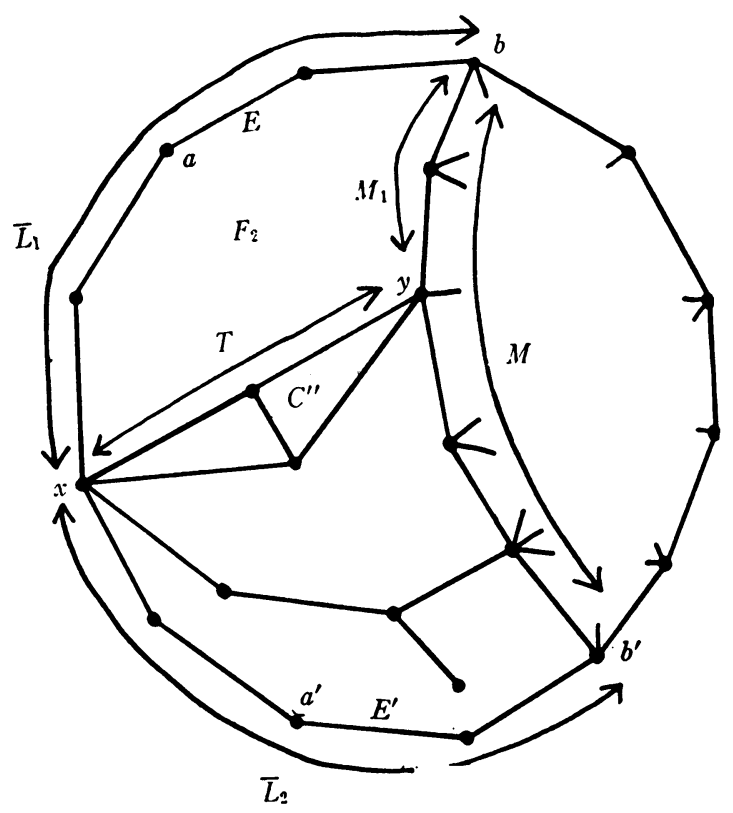

FIG. VI

enclosed by $B_{i}$. It would therefore not be a subset of $G_{i}$. Accordingly $y$ separates $M$ into two disjoint arc-graphs, $M_{1}$ with ends $b$ and $y$ and $M_{2}$ with ends $y$ and $b^{\prime}$.

Let $F$ be the residual domain of the circuit $\bar{L}_{1} \cup T \cup M_{1}$ contained in $R_{2}$. Then $F_{2} \subseteq F \subseteq F_{4}$. But if an edge $A$ of $M^{\prime}$ meets $F$ it is an edge of a transversal $M^{\prime \prime}$ of $P_{4}$ over $F_{4}$ whose ends are vertices of $M$. This is impossible by (2.2) and (4.1). We deduce that $F=F_{2}$. Hence $P_{2}=\bar{L}_{1} \cup T \cup M_{1}$.

We choose $U^{\prime \prime}$ as an edge of $M$ incident with $y$. Then $y$ is a vertex of $Q_{i}$. Hence $C^{\prime \prime}$ is a bridge of $Q_{i}$. Since $w\left(C^{\prime \prime}\right)=2$ it satisfies (ii).

Let $C$ be any bridge over $S_{2}$ in $G_{i}$ such that $q\left(C, Q_{i}-\bar{L}\right) \geqq 1$, that is, having $x$ as a vertex. Applying (2.4) we find that there is a bridge $B$ over $F_{4}$ in $G_{i}$ which is a subset of $C$ and which has $x$ as a vertex. Then $w(B) \leqq 2$ by (4.1). If each vertex of attachment of $B$ is a vertex of $Q_{i}$ we have $B=C$. Then $C$ satisfies (ii) since $B$ meets $P_{2}=\bar{L}_{1} \cup T \cup M_{1}$ only if $B=C^{\prime \prime}$. Suppose however that $B$ has a vertex of attachment $z$ which is not a vertex of $Q_{i}$. If $z$ is an internal vertex of $M_{1}$ then $|B|$ meets $F_{2}$. It follows that $B$ is a bridge over $F_{2}$, by the corollary to (2.4). But this is impossible by $(4.1)$ since $w(B)=2$. We deduce that $z$ is an internal vertex of $M_{2}$. Then $C$ meets $M_{2}$ and so $p(C$, $\left.Q_{i}-\bar{L}\right)=2$, by (2). Hence $w(C)=3$. Further the three vertices of attachment of $C$ must be $x$ and two vertices of $M_{2}$. There can be no further vertex of attachment of $C$ which is a vertex of $M_{1}$ and therefore $C$ does not meet $M_{1}$. 
Since $C$ is not $C^{\prime \prime}$ it follows that $C$ does not meet $P_{2}$ and therefore does not meet $K_{2}$. Again condition (ii) is satisfied.

By Lemma I there remains only the case in which $p\left(B_{i}, Q-L\right)=2$. In this case we denote the common vertices of $B_{i}$ and $Q-L$ by $x$ and $y$ so that $x$ separates $b$ and $y$ in $\bar{L}$. Then $x$ and $y$ are the only vertices of $Z_{i}$ which are internal vertices of $\bar{L}$.

A vertex $t$ of $M$ is $x$-linked ( $y$-linked) if there is a transversal of $P_{4}$ over $F_{4}$ in $G_{i}$ with ends $x$ and $t(y$ and $t)$. Now if $t$ is an $x$-linked and $u$ a $y$-linked vertex of $M$ then $t$ and $x$ do not separate $u$ and $y$ in $\left|P_{4}\right|$ by (4.1). Further $M$ has at least one internal vertex. For otherwise $M$ would be a bridge of $Q$ in $G$ distinct from $B_{i}$ and not enclosed by $B_{i}$, contrary to the definition of $G_{i}$. It follows that there is an internal vertex $z$ of $M$ separating $M$ into two disjoint arc-graphs, $M_{1}$ with ends $b$ and $z$ and $M_{2}$ with ends $z$ and $b^{\prime}$, in such a way that all the $x$-linked vertices of $M$ are vertices of $M_{1}$ and all the $y$-linked vertices of $M$ are vertices of $M_{2}$. (See Figure VII.)

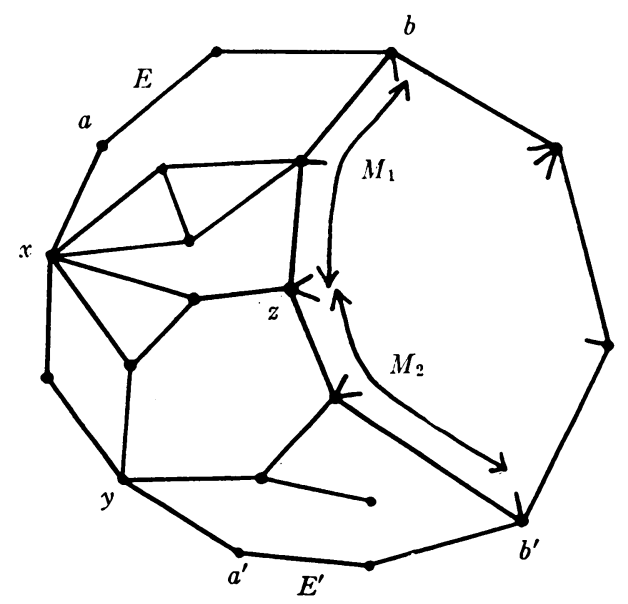

FIG. VII

Having found such a vertex $z$ we choose $U^{\prime \prime}$ to be incident with $z$. Then $z$ is a vertex of $Q_{i}$.

Let $C$ be any bridge over $S_{2}$ in $G_{i}$ such that $q\left(C, Q_{i}-\bar{L}\right) \geqq 1$. Then either $x$ or $y$ is a vertex of $C$. If $x$ is a vertex of $C$ we find by (2.4) that there is a bridge $B$ over $F_{i}$ in $G_{i}$ which is a subset of $C$ and has $x$ as a vertex. Then $w(B) \leqq 3$ by (4.1). If each vertex of attachment of $B$ is a vertex of $Q_{i}$ we have $B=C$. If instead $B$ has a vertex of attachment $t$ which is not a vertex of $Q_{i}$ then $t$ is an internal vertex of $M_{1}$, since it is an $x$-linked vertex of $M$ by (2.1). Hence either $w(C) \leqq 3$ or $M_{1}$ and $C$ have a common edge. Similarly if $y$ is a vertex of $C$ we find that either $w(C) \leqq 3$ or $C$ meets $M_{2}$. 
Assume $w(C) \geqq 4$. Then $C$ meets either $M_{1}$ or $M_{2}$ and therefore $p(C$, $\left.Q_{i}-\bar{L}\right)=2$ by (2). Accordingly both $x$ and $y$ must be vertices of $C$. But then $C$ meets both $M_{1}$ and $M_{2}$. Hence at least two vertices of $M_{1}$ and at least two vertices of $M_{2}$ are vertices of attachment of $C$. This is impossible since $p\left(C, Q_{i}-\bar{L}\right)=2$ and the arc-graphs $M_{1}$ and $M_{2}$ have only one common vertex.

We deduce that $w(C) \leqq 3$. Now each edge of $C$ incident with $x$ or $y$ must belong to $B_{i}$, by the definition of $G_{i}$. But $B_{i} \cap C$ is $Q_{i}$-bounded since $C$ does not meet $Q$ (because $S_{2} \subseteq R_{2}$ ). Hence $B_{i} \cap C=C$ and so $C \subseteq B_{i}$. Hence by Lemma I, $C$ does not meet $K_{2}$. Accordingly $C$ satisfies (ii).

We have now verified that if $U^{\prime \prime}$ is suitably chosen the circuit $Q_{i}$ must satisfy condition (ii). The lemma now follows from (1).

We obtain a planar graph $J$ from $Q$ by replacing the arc-graph $L\left(B_{i}\right)$ by the arc-graph $Q_{i}-\bar{L}$ in each of the singularities $Z_{i}$. It is easily verified that $J$ is a circuit of $G$ having all the vertices of attachment of the singularities $Z_{i}$ and the norm $Z$ of $Q$ as vertices.

Let $B$ be any bridge of $J$ in $G$. Then $B$ meets $W$, where $W$ is either $Z$ or one of the singularities $Z_{i}$ of $Q$. In each case $W \cap B$ is $J$-bounded, whence it follows that $B \subseteq W$. If $W=Z$ this means that $B$ is a nonsingular bridge of $Q$ in $G$. Then $w(B) \leqq 3$, and $w(B)=2$ if $B$ meets $K_{1}$ or $K_{2}$. If $W=Z_{i}$ then $B$ is a bridge of $Q_{i}$ in $G_{i}$ and we apply Lemma II to obtain the same result.

It follows that Theorem I is true for the planar graph $G$. Since $G$ may be any member of $\boldsymbol{Y}$ we have $\boldsymbol{Y} \subseteq X$, as required.

Theorem I now follows by induction. For we have seen that it is trivially true if $\alpha_{1}(G) \leqq 1$. And if it is true for all planar graphs with $n$ or fewer edges it is also true for those with $n+1$, since such graphs belong to $Y$ and therefore, by $(5.2)$, to $X$.

6. Hamiltonian circuits. Using (3.2) and (4.1) we see that if $K$ is a terminal circuit of an edge $E$ of a planar graph $G$ and if $E^{\prime}$ is another edge of $K$ then $K$ is also a terminal circuit of $E^{\prime}$. Accordingly we speak of the terminal circuits of the edges of $G$ simply as the terminal circuits of $G$.

THEOREM II. Let $G$ be any 4-connected planar graph having at least two edges. Then $G$ has a Hamiltonian circuit. Moreover if no two edges of $G$ have both ends in common, and if $E$ and $E^{\prime}$ are distinct edges of the same terminal circuit of $G$, then there is a Hamiltonian circuit of $G$ having both $E$ and $E^{\prime}$ as edges.

Proof. We suppose first that no two edges of $G$ have both ends in common. Let $E$ and $E^{\prime}$ be distinct edges of a terminal circuit $K$ of $G$. There is a circuit $J$ of $G$ satisfying conditions (i), (ii) and (iii) of Theorem I. If each bridge of $J$ in $G$ has just one edge, of which both ends are vertices of $J$, then $J$ is a Hamiltonian circuit of $G$ having $E$ and $E^{\prime}$ as edges. If not it follows from (3.5) and the restriction on $G$ that $\alpha_{1}(J)=3$, that there is only one bridge of $J$ in $G$, and that this bridge has attachment-number 3. But by (4.3) the 
bridge must meet a terminal circuit of $E$. This is impossible by condition (iii) of Theorem I.

To prove the first part of the theorem we observe that $G$ has at least one circuit, by (3.3). If $G$ has only two vertices each circuit of $G$ is a Hamiltonian circuit. Accordingly we assume $\alpha_{0}(G) \geqq 3$. If we remove some edge of $G$ which has the same ends as some other we obtain from $G$ another 4-connected planar graph with the same vertices. Repeating this operation sufficiently of ten we obtain a 4-connected planar graph $H$ in which no two edges have both ends in common, and which has the same vertices as $G$. Since $\alpha_{0}(G) \geqq 3$ we have $\alpha_{1}(H) \geqq 2$. Hence by (3.3) $H$ has a circuit and therefore a terminal circuit. So by the result already proved $H$ has a Hamiltonian circuit $J$. This is also a Hamiltonian circuit of $G$.

Hassler Whitney's theorem [2] corresponds to the special case of Theorem II in which each terminal circuit has just three edges.

7. A note on the four colour conjecture. It is a commonplace of the theory of map-colourings that any map defined by a planar graph having a Hamiltonian circuit can be coloured in four colours. The regions in one residual domain of the circuit can be coloured alternately red and blue and those in the other alternately green and yellow. So we can now assert that the Four Colour Conjecture is true for all maps defined by 4-connected planar graphs.

Unfortunately the maps of most interest are defined by planar graphs in which the degree of each vertex is 3 . If such a planar graph has 5 or more vertices it is not 4-connected, by (3.4). Little is known about conditions for the existence of a Hamiltonian circuit in a planar graph $G$ of this kind, except that it is not sufficient for $G$ to be 3-connected [1].

\section{REFERENCES}

1. W. T. Tutte, On Hamiltonian circuits, J. London Math. Soc. vol. 21 (1946) pp. 98-101.

2. Hassler Whitney, A theorem on graphs, Ann. of Math. vol. 32 (1931) pp. 378-390.

UNIVERSITY OF TORONTO,

TORONTO, ONTARIO. 